\title{
Vitamin Transport Diseases of Brain: Focus on Folates, Thiamine and Riboflavin
}

\section{Reynold Spector}

Department of Medicine, Robert Wood Johnson Medical School, 105 Stone Hill Road, Colts Neck, NJ 07722, USA

*Corresponding author: Reynold Spector, Adjunct Professor of Medicine, Department of Medicine, Robert Wood Johnson Medical School, 105 Stone Hill Road, Colts Neck, NJ 07722, USA, Tel: 732-780-5762; E-mail: mspec007@verizon.net

Received date: Apr 2, 2014, Accepted date: Apr 24, 2014, Published date: Apr 30, 2014

Copyright: (C) 2014 Spector R. This is an open-access article distributed under the terms of the Creative Commons Attribution License, which permits unrestricted use, distribution, and reproduction in any medium, provided the original author and source are credited.

\begin{abstract}
Background: In the last decade, childhood neurological disorders involving inadequate transport of thiamine, riboflavin or folates into brain have been defined. These involve two transporters of folate (the folate receptor $\alpha$ or the proton-coupled folate transporter), one of thiamine and two of the six riboflavin transporters. Depending on the mutations involved, without a specific diagnosis and treatment, the phenotypes vary from mild to severe and fatal.
\end{abstract}

Methods: The purpose of this review is to summarize the relevant physiology and molecular biology before describing the clinical (neurological) manifestations of these either homozygous or compound heterozygous autosomal recessive disorders. In all cases (but one due to easily measurable antibodies to the folate receptor $\alpha$ transporter), the diagnosis can be apodictically confirmed by DNA sequencing of the appropriate genes.

Results: With a definitive diagnosis, appropriate ultra-high dose vitamin therapy can be instituted, and, if done before irreversible damage has occurred, can ameliorate or cure the patient. However, treatment is sometimes only marginally successful, in part, because of irreversible brain damage, but also because current oral therapy may not deliver enough of the appropriate vitamin to brain. Approaches to further improve therapy are discussed.

Conclusions: In summary, a group of previously undefined childhood vitamin transport neurological disorders have been identified, understood and can be diagnosed on a molecular level. Moreover, this has led to rational and in some cases effective treatment, a triumph of modern pediatric neurology.

Keywords: Autosomal Recessive; Vitamins; Mutations; Vitamin Transporters; Blood-Brain Barrier.

\section{Introduction}

Almost one hundred years ago, medical scientists recognized that several devastating brain disorders were due to lack of micronutrients e.g., Wernicke's encephalopathy. Inclusion of certain foods or later specific vitamins prevented or cured these disorders if irreversible brain damage had not occurred. But exactly how, and why these nutrients and vitamins "worked" has only been understood gradually over the last fifty years. One critical point was how do vitamins enter CSF and brain from blood and what are the kinetics [1]. This question arose with the discovery of the blood-brain barrier (BBB), consisting of the tight junctions connecting the cerebral capillaries and the bloodCSF barrier (BCSFB), the choroid plexus (CP) epithelial cells (which secrete the CSF) and the multi-layered arachnoid membrane - both joined by tight junctions between the cells [1]. With this anatomical understanding came the realization that water-soluble molecules the size of vitamins could not simply diffuse through the BBB and BCSFB but there must be specific transport mechanisms [1]. Moreover, there was $\sim 4$ times more methyltetrahydrofolate (MeTHF) and ascorbic acid (AA) in CSF than plasma [1-4]. (MeTHF is the principal plasma and CSF folate.) This suggested there were active transport systems at work [2-5]. Finally, when studied, investigators found that the brain was generally the last organ to be depleted of vitamins in severe deficiency states $[1,5]$. We now know the reason: For the three vitamins discussed in this paper, folates, riboflavin (R) and thiamine
(T), there are separate specific transport (homeostatic) systems at the $\mathrm{BBB}$ and/or BCSFB that help maintain constancy of these vitamins in brain and CSF [1,5-7]. These transport systems do this, in part, because the plasma vitamin concentration is $\sim$ equal to the halfsaturation concentration of these carrier-mediated transport systems [1,5-7]. So when the blood level is low, relatively more vitamin is transported into brain; when high, relatively less. Moreover, there are also separate specific saturable transport systems in brain cells including neurons that accumulate folates, $\mathrm{R}$ and $\mathrm{T}$ from the CSF and extracellular space (ECS) of brain [1-7]. These cellular transport systems are also $\sim 1 / 2$ saturated at the normal CSF/ECS concentration [1-7]. Thus, the brain barrier transport and, in series, the cellular transport systems (on the other side of the BBB and BCSFB), provide impressive control of entry of the transported vitamer (the moiety of the vitamin transported) into brain cells. Moreover, within the brain cells there are mechanisms that regulate the amount of vitamin in the cell. It should be noted that vitamin homeostasis in brain is not due to lack of turnover. In fact, in brain, $\mathrm{T}$ and $\mathrm{R}$ turn over relatively rapidly; folates very substantially slower [3-7]. However, when these dynamic transport/homeostatic systems fail, e.g., due to genetic defects, the brain becomes deprived of that vitamin. For example, in rabbits which turn out to be a reasonable model for humans, less than $5 \%$ of total brain T, R and folate enter brain by simple diffusion [4-7].

Gradually, over the last decades, with careful physiological, pharmacological and biochemical studies in animals and humans, and more recently the use of molecular biological techniques such as cloning and knock-outs $(\mathrm{KO})$ in mice and rats, it was proven, first in rodents and later in humans, on a molecular level that there were 
indeed multiple separate specific vitamin transport systems at the BBB and/or BCSFB for individual vitamins with one exception as documented below. (There is one system that transports biotin, pantothenic and lipoic acids at the BBB) [1]. As these systems were described and understood, careful clinical research documented there were patients with heretofore unrecognized neurological disorders involving these CNS transport systems, some of which were devastating or even fatal. Once understood, in some cases, methods of diagnosing and treating these disorders became available.

The purpose of this review is to focus on the autosomal recessive CNS diseases of folate, thiamine and riboflavin transport into brain because there is new information on diagnosis and treatment. In each case, after a brief background, I will describe the biology of the transporters, the associated clinical syndromes, and the methods of diagnosis and treatment. Because in a short review it is not possible to be comprehensive, I will refer to the most recent work - focusing on the last several years.

To aid in explanatory power, I will first focus on what used to be termed "genetic penetrance" which can now be understood on a molecular basis. In the autosomal recessive transport disorders described below (with one exception) for folates, $\mathrm{R}$ and $\mathrm{T}$, there are mutations in each chromosomal transport gene, sometimes the same, sometimes different (i.e., homozygous or compound heterozygotes.) In fact, there are examples of deletions, frame shifts, nonsense or missense mutations. Some of these do not affect gene transcription or translation. However, some prevent synthesis of messenger RNA or the proper translocation of the transporter to the appropriate place on the membrane or the function of the transporter. The severity of the disorder in an individual patient depends on the specific nature of the mutations - thus explaining the notion of "penetrance" i.e., the variability of signs and symptoms and age of onset. Of course, in these vitamin transport disorders, physicians generally diagnose only those persons with deleterious mutations in both chromosomes - called autosomal recessive inheritance. Second, it is clear the $\mathrm{KO}$ animals and patients with these disorders can be studied and the exact mutations found by DNA sequencing. The utility of this approach is clear. Moreover, in a proband whose DNA has been sequenced, the DNA of the siblings and/or children can also be sequenced. If positive, appropriate treatment, in some cases, can be instituted, thus preventing devastating and irreversible neurological damage. (See below). Third, it is clear that in some cases both in humans and $\mathrm{KO}$ mice, the $\mathrm{KO}$ of one pair of transport genes may be compensated for by existing systems as described in several cases below. Fourth, in some disorders, normal vitamin blood levels do not predict CNS or other bodily disorders. Finally, there are specialized transporters within cells that transport MeTHF, flavin adenine dinucleotide (FAD) and thiamine pyrophosphate (TPP) into mitochondria [8-10]. I will not discuss the FAD and folate mitochondrial transporters since there are no known human disorders caused by their lack [8,9]. However, although a mouse KO of the TPP transporter (slc25a19) into mitochondria is embryonically lethal, an apparently less severe, autosomally recessive mutation in the human analogue SLC25A19 is responsible for Amish lethal microcephaly [10].

\section{Folates in CSF and Brain}

In brain, various folates play well known essential roles in brain metabolism. For example, reduced folates (in various methylated forms) are the cofactors in the methylation of homocysteine to methionine [11,12] and the formation of thymidine from deoxyuridine via S-adenosylmethionine [13]. Within cells reduced folates are polyglutamated; however, only the monoglutamated forms are transported across cell membranes [1].

In both humans and rabbits, folates, after hydrolysis of polyglutamates to monoglutamate forms, are absorbed in the gastrointestinal tract by a mechanism that depends on the protoncoupled folate transporter (PCFT; SLC46A1) [14]. This transporter is on the apical (luminal) surface of intestinal epithelia [14]. Exactly how folates exit the intestinal epithelia into the blood remains uncertain, possibly by the reduced folate carrier (RFC; SLC 19A1) on the abluminal side [14]. Once transferred into blood, the concentration of MeTHF $(\sim 20 \mathrm{nM})$ is normally maintained relatively constant by saturable mechanisms in the both the gastrointestinal tract and kidney $[5,14]$. From the blood, MeTHF enters the CSF by a complex system in $\mathrm{CP}$ involving the folate receptor $\alpha(\mathrm{FR} \alpha)$, PCFT and RFC. There is substantial evidence that FRa binds plasma MeTHF on the blood side (basal surface) of the CP and enters endosomes [3,4,14]. MeTHF is then released from the FRa within endosomes by PCFT and exits the CP epithelial cells into CSF probably by RFC $[14,15]$. This complex system in $\mathrm{CP}$ is half-saturated at $\sim 20 \mathrm{nM}$ and thus at low plasma concentrations pumps more MeTHF into CSF and at high plasma concentrations less, thus providing a crucial part of the homeostatic system for folates in CSF $[5,15]$. From CSF, folates gradually enter brain cells presumably by RFC on cellular surfaces. There appears to be no FRa at the BBB and no or minimal transport of folates through the BBB $[14,15]$.

Homozygous KO mice lacking FRa, PCFT or RFC are all embryonically lethal or die soon after birth [16-18]. But if heterozygous dams for the mutation are supported with massive doses of folate, some viable homozygous mice (autosomal recessive) lacking the specific transporter can be delivered and kept alive with high doses of folates [16-18].

Homozygous or compound heterozygous humans with abnormalities of the PCFT transporter have been described [19]. The presentation is variable depending on the exact mutations but in general these infants are normal at birth but develop anemia, infections due to immune deficiency and later developmental delays, ataxia and seizures recently termed cerebral folate deficiency (CFD) [19]. As expected, they have both low blood MeTHF and very low CSF MeTHF since PCFT is involved in transport of MeTHF across the intestine and choroid plexus into CSF; from CSF, Me THF is then accumulated by brain cells $[14,15,19]$.

A second related autosomal recessive syndrome is due to a deficiency of FRa [20-22]. Generally these patients present one to two years after birth, depending on the mutations in the FR genes. Like PFCT deficient patients, they often present with CFD including seizures, delayed motor development, ataxia, decreased head growth and other abnormalities including myoclonus and white matter changes [20-22]. As expected, these patients have a normal blood folate concentration but low or very low CSF folate concentrations [20-22].

A third folate transport syndrome (CFD) with low CSF folates is due to the development of antibodies to FRa [23]. Unlike PFCT and FR $\alpha$ disorders, the antibody syndromes have myriad presentations including infantile developmental disorders, infantile neurological abnormalities with autism, infantile autism, spastic-ataxia after age one, and at a later age dystonia progressing to bradykinesia and finally a pyramidal syndrome occurring in adolescents to young adulthood. 
There are however some confusing aspects to these antibody syndromes although they all have in common low CSF folate [23]. For example, over a period of several weeks FRa blocking antibodies may go from high to undetectable [23]. Second, antibodies may be IgG or IgM [23]. Finally, there is no definitive MRI or CAT scan finding although on MR spectroscopy there may be loss of inositol and choline peaks in white matter which also occurs in FRa mutations [23].

The diagnosis of CFD due to autosomal recessive abnormalities of PCFT and FRa, or blocking antibodies to FRa can be strongly suspected in patients with a decreased CSF folate concentration and, in the case of PCFT mutations, also in blood. Of course, several other genetic conditions can cause decreased CSF folate e.g., the KearnsSayre syndrome and dihydrofolate reductase deficiency [24,25]. Definitive diagnosis can be made by sequencing the suspect genes on both chromosomes thus providing an exact diagnosis in the case of PCFT and FR $\alpha$ mutations. This is critical so that family members and children can be properly counseled. In the case of suspected causal FRa auto-antibodies, Ramaekers et al. propose a systematic evaluation including of course measurement of CSF folate and if low, anti-FRa antibodies [23].

For treatment, it is crucial to make an accurate diagnosis of CFD as soon as possible (to avoid irreversible brain damage) beginning with measuring blood and CSF folates. Therapy with massive doses of folinic acid (1 to $5 \mathrm{mg} / \mathrm{kg} /$ per day) can reverse the disease processes or at least stabilize these patients who otherwise generally continue to progress and sometimes die [19-25]. It should be noted that these patients are unlike otherwise normal patients with nutritional folate deficiency who respond to Recommended Daily Allowance (RDA) amounts of folate, because in all three syndromes (caused by PCFT or $\mathrm{FR} \alpha$ mutations or FR $\alpha$ antibodies) these patients lack the mechanism to transport MeTHF from blood into CSF via CP which secondarily leads to insufficient folate available to brain. Thus they require massive doses of folate to overcome the transport deficiency [14-23]. When high dose folate therapy is instituted, enough folate enters CSF and brain (by uncertain mechanisms) in many patients to carry out the myriad reactions in which folate is a cofactor, e.g., the production of methionine from homocysteine and the synthesis of thymidine from deoxyuridine by thymidylate synthetase [15]. However, it is not clear what concentration of CSF folate should be the target. Is just normalization of the CSF folate adequate or would a higher concentration result in better clinical outcomes? A higher CSF concentration could almost certainly be obtained by continuous intravenous MeTHF. See below for further discussion of vitamin treatment objectives, i.e., surrogate markers versus clinical outcomes. Finally, in the case of antibody-induced CFD, there is anecdotal evidence that consumption of milk by these patients exacerbates antibody formation because milk contains small amounts of FR [23]. Ramaekers et al. suggest it is prudent to stop milk consumption although there is no convincing clinical evidence that this practice is beneficial [23].

\section{Thiamine}

As is well known, T as TPP is an essential cofactor for multiple critical enzymatic reactions in brain. Lack of $\mathrm{T}$ in diet, especially when coupled with alcohol abuse or certain disorders of the GI tract, can lead to the Wernicke-Korsakoff syndrome or heart dysfunction (beriberi). These deficiency disorders can be reversed by $\mathrm{T}$ if irreversible damage has not occurred, e.g., Korsakoff's psychosis. There are also several CNS enzymatic disorders that employ TPP and, as described below, transport disorders [26-28]. T occurs in brain cells mainly as TPP but there are also small amounts of T itself, T. monophosphate (TMP) and the triphosphate (TTP) [26-29].

In humans and rodents, there are three thiamine transporters SLC 19A1, A2, and A3 [19] as well as the separate transporter that transports TPP into mitochondria [30]. In humans and rodent plasma and CSF, $\mathrm{T}$ is present as free T and TMP [26-29]. In our early studies of $\mathrm{T}$ transport across the rabbit $\mathrm{BBB}$ and $\mathrm{BCSFB}$, we found that $\mathrm{T}$ was transported through the BBB by a saturable system with a one-half saturation concentration (KT) of $\sim 0.2 \mu \mathrm{M}$ [6]. This was later confirmed in rats $[31,32]$. Subsequently, based on a suggestion by us, Rindi's group provided evidence that TMP could be transported into brain but much less efficiently with a KT $\sim 2.0 \mu \mathrm{M}[26-29,33]$. The plasma and CSF total $\mathrm{T}$ concentrations in humans are $\sim 0.1 \mu \mathrm{M}$ with the majority TMP and the remainder T [26-29,33,34].In rabbits, the $\mathrm{CP}$ actively accumulates $\mathrm{T}$ and releases both $\mathrm{T}$ and TMP into CSF, thus explaining the origin of TMP in CSF which exceeds that of T $[6,33]$. Brain cells accumulate $\mathrm{T}$ by facilitated diffusion $(\mathrm{KT} \sim 0.3 \mu \mathrm{M})$ and intracellular trapping of TPP by $\mathrm{T}$ pyrophosphokinase [6]. Subsequent elegant molecular biological work including cloning of the human transporters showed that SLC 19A1, the RFC transporter discussed above, not only transports MeTHF and but also TMP (but not T), with affinities for TMP consistent with in vivo data [19]. It is worth emphasizing that the RFC (SLC19A1) is especially prominent on the apical side of $\mathrm{CP}$, as noted above, and thus in a position to release TMP into CSF [6,26-29,33]. Similarly, neurons that contain RFC can also take TMP from CSF although presumably less effectively than $\mathrm{T}$.

SLC 19A2 (19A2) and SLC 19A3 (19A3) transport only T [19]. In humans, the thiamine transporter 19A2 appears on the abluminal side of intestinal cells, on red cell membranes, and on the luminal (blood) side of brain capillaries [35,36]. The KT of the facilitated diffusion system of 19A2 in human red cells is $\sim 0.3 \mu \mathrm{M}[19,34]$. Unlike 19A2, $19 \mathrm{~A} 3$ resides on the apical (luminal) side of intestinal epithelial cells and renal tubular cells, and surprisingly on the albuminal side of human cerebral capillaries [19,35,36].

There are two different human syndromes associated with $\mathrm{KO}$ of 19A2 and 19A3, respectively. Autosomal recessive knock out of 19A2 in humans is known as thiamine-responsive megaloblastic anemia (TRMA) consisting of anemia, diabetes and deafness. Plasma thiamine levels are normal [34]. TMRA anemia is understandable since $\mathrm{T}$ transport into red cells depends on 19A2 [34]. High dose $\mathrm{T}$ is employed to treat this disorder which can be diagnosed definitively by DNA sequencing. However, except for deafness, there is no CNS involvement and I will not further discuss this syndrome. However, it should be noted that 19A1 (which transports TMP) and 19A3 are able (without 19A2) to supply the plasma and brain with adequate amounts of $\mathrm{T}$ [34].

Human autosomal recessive KO of 19A3 leads to several CNS syndromes proven by the elegant work of multiple investigators. The most severe form occurs in early-infancy and is a form of lethal encephalopathy [36]. A less severe syndrome named thiamineresponsive basal ganglia (TBGD) disease occurs later in childhood [37]. Third, in the second decade of life a Wernicke-like syndrome has been reported [36,37]. There is substantial variability in these cases but all show homozygous or compound heterozygous autosomal recessive inheritance. 
The clinical diagnosis of these 19A3 disorders depends on MRI changes in the putamen-caudate in the more severe cases, elevation of serum lactate due to energy failure and response to high doses of thiamine if treatment is initiated before irreversible damage occurs $[36,37]$. There is not enough data on plasma and CSF levels of $T$ and TMP at this time to be too helpful. The definitive diagnosis depends on DNA sequencing of 19A3 [36,37]. Once again, it is extremely important to prove the diagnosis so that siblings and children of the proband can be rationally studied and if necessary treated. There have been cases of as yet unaffected children who are homozygous for deleterious 19A3 mutations, who when treated early, are normal $[36,37]$.

If the diagnosis is made, treatment with high doses of $\mathrm{T}$ is able to stabilize or even reverse brain dysfunction especially in TBGD.37 In one series of TBGD cases, four patients that presented with encephalopathy were rendered normal with high dose oral thiamine therapy (100-300 mg/day) [37].

\section{Riboflavin}

In brain, riboflavin as flavin mononucleotide (FMN) and FAD serve as co-factors in many crucial enzymatic reactions [7]. In human and rabbit plasma, total riboflavin $(\mathrm{R}+\mathrm{FMN}+\mathrm{FAD})$ is $\sim 0.2 \mu \mathrm{M}$ with $\sim$ $0.05 \mu \mathrm{M} \mathrm{R}$, the remainder FMN and FAD [7,38-41]. In CSF, total riboflavin is $\sim 0.1 \mu \mathrm{M}$ with $\sim 0.02 \mu \mathrm{M} \mathrm{R}[7,38-41]$.

There are six riboflavin transporters I will mention as part of my description of the physiology. As noted above, FAD is transported into mitochonodria but there are no known neurological disorders associated with this transporter [8]. Second, breast cancer resistance protein (ABCG2) is involved in the transfer of $\mathrm{R}$ into breast milk, but a $\mathrm{KO}$ of this protein is inconsequential because $\mathrm{R}$ (as FAD) enters breast milk by another route [42]. The third transporter that vigorously transports $\mathrm{R}$ is the "efflux" organic acid transporter 3 (OAT3) in kidney and choroid plexus $[7,38]$. See below. Finally, there are the three recently discovered, cloned and characterized human $\mathrm{R}$ transporters SLC52 A1, A2, A3 (52A1, 52A2, 52A3) [43-45].

Riboflavin transport physiology in humans began in earnest when Jusko and collaborators unraveled $\mathrm{R}$ handling by the kidney and intestine [46]; they showed in humans that $\mathrm{R}$ is normally almost completely reabsorbed from the glomerular filtrate (GFR) by a saturable system in the renal tubules. But if a concentration of $>0.5$ $\mu \mathrm{M} R$ in the GFR occurred, the system was saturated and $\mathrm{R}$ spilled into the urine. We now know that the reabsorption transporter on the apical side of the tubule (and intestine) is 52A3 [47,48]. Moreover, above $0.5 \mu \mathrm{M}$ R in plasma, we also know that OAT-3 (on the basal side of the renal tubule) secretes excess $\mathrm{R}$ (and drugs like penicillin) into the urine $[39,46]$. Unlike the $\mathrm{R}$ transporters $52 \mathrm{~A} 1,52 \mathrm{~A} 2$, and $52 \mathrm{~A} 3$ which have KT's below $1 \mu \mathrm{M}$ [48], OAT-3 has a KT $\sim 75 \mu \mathrm{M}[38,39]$.

In the intestine, there is now very strong evidence that $52 \mathrm{~A} 3$, on the luminal side, and $52 \mathrm{~A} 1$ on the abluminal side of the epithelial cells, absorbs R from the intestinal lumen into theblood[46-48], FAD and FMN are hydrolyzed to $\mathrm{R}$ before absorption [47]. Together these saturable systems in gut and kidney tend to keep total plasma $\mathrm{R}$ levels constant $[48,46]$. As noted above, similar systems in kidney and intestine tend to keep $\mathrm{T}$ and folate plasma levels constant.

$\mathrm{R}$ enters brain from blood at the BBB by a saturable system (KT $0.1 \mu \mathrm{M})$ dependent on 52A2 [7,48-53]. From the extracellular space of brain, brain cells accumulate $\mathrm{R}$ by facilitated diffusion (presumably by
52A2) and rapidly convert the $\mathrm{R}$ to FMN and FAD [7,41]. Total riboflavin (mainly FMN and FAD) in rabbit brain $(\sim 10 \mu \mathrm{M})$ turns over by $\sim 50 \%$ in several days [7]. One great surprise was the finding that the CP via OAT3 rapidly clears $\mathrm{R}$ out of CSF [7,39]. In fact 2 hours after the intraventricular injection of tracer concentrations of $\mathrm{R}$ and mannitol into CSF of unanesthetized rabbits, there is only $2 \%$ as much $\mathrm{R}$ in CSF as the passively distributed mannitol [7,39]. There is as yet no physiological explanation for the rapid active efflux of $\mathrm{R}$ from CSF into blood via OAT3 in CP.

There are two separate but related human syndromes associated with homozygous or compound heterozygous autosomal recessive knock outs of 52A2 and 52A3 [48-53]. In patients with 52A2 mutations, the more seriously affected children manifest a phenotype of a progressive axonal sensorimotor neuropathy, hearing loss, optic atrophy and respiratory insufficiency [48-53]. In the past this has been called the Brown-Vialleto-Van Leare (BVVL) syndrome. As expected, these patients have normal total riboflavin concentrations in blood and normal brain MRI's. The problem is that $\mathrm{R}$ cannot enter brain from blood because of mutations in $52 \mathrm{~A} 2$ which is essential for $\mathrm{R}$ transport into brain through the BBB [48-53]. Although there are often changes in urinary organic acids and elevated plasma acylcarnitine [49], the definitive diagnosis of the BVVL syndrome can be made based on analysis of the mutations in the 52A2 genes after DNA sequencing. The CSF concentrations of $\mathrm{R}$ is not helpful because of the rapid removal of R by OAT 3 as described above $[7,39]$.

Treatment consists of high dose 10 to $40 \mathrm{mg} / \mathrm{kg}$ per day oral riboflavin [49-53]. In some cases, this causes stabilization or improvement of the signs and symptoms of the disorder. However, one problem, even with very high oral doses of $\mathrm{R}$, is the homeostatic systems (in intestine and kidneys discussed above) tends to keep total $\mathrm{R}$ in blood fairly constant militating against big increases in total $\mathrm{R}$. For example, in eight BVVL patients due to 52A2 mutations, the average blood concentration of FAD before therapy was normal $(0.27$ $\mu \mathrm{M} ; \mathrm{N}=8$ ) [53]. There was not enough data on $\mathrm{R}$ itself to comment. After high dose oral therapy there was only a $35 \pm 7 \%(\mathrm{~N}=8$; SEM) increase in blood (plasma) FAD [53]. I hypothesize that to drive R into brain by simple diffusion i.e., to bypass the lack of $52 \mathrm{~A} 2$ at the BBB, one would have to consider constant intravenous infusions of R. (See below)

A similar syndrome but with a different pathophysiology consists of autosomal recessive mutations in the 52A3 gene [49,51]. As expected, these children (unlike those with 52A2 mutations) have low plasma concentrations of R, FMN and FAD [49,51]. This diagnosis is confirmed by sequencing of the $52 \mathrm{~A} 3$ genes $[49,51]$. The treatment of patients with $52 \mathrm{~A} 3$ mutations is also high dose R. The few reported treated patients have responded and, in a few cases, normalized their plasma R, FMN and FAD [49,51]. However, there are too few reported cases of 52A 3 mutations to be certain of exact benefit with high dose riboflavin therapy, but in several cases, there have been very impressive responses [49]. Early diagnosis and treatment with R before irreversible neurological damage is done should greatly benefit such patients since we now understand the pathophysiology (and potential treatment) in patients with $52 \mathrm{~A} 3$ mutations.

\section{Comment}

The history of the discovery of the vitamin transport diseases of brain sheds light on the proper diagnosis and treatment of these disorders, and where crucial knowledge is incomplete. First, the 
anatomists found and described the BBB and BCSFB, which would prohibit entry of water-soluble vitamins unless there were transport systems. Then, the physiologists and biochemists discovered and described the systems in animals and humans with emphasis on the specificity and kinetics of the systems. Next, the molecular biologists and anatomists found, cloned, localized and characterized the transporters individually. There is remarkable coherence in all this work (except for differences between some $\mathrm{KO}$ mice and human autosomal recessive diseases. (See below.) Finally clever neurologists uncovered the various disorders described above. This historical sequence shows the power of brain research to find (and treat) previously crippling if not fatal diseases in the proband and, with further genetic testing in appropriate family members, those at risk for disease. Also, I suspect the small numbers of patients so far described are just the tip of the clinical iceberg, as there are probably many persons with milder forms and less severe genetic mutations.

There are however problems in diagnosing some of these disorders and understanding their manifestations, in part, due to genetic heterogeneity. For example, in human thiamine SLC 19A3 KO's, there is tremendous variability in age of onset (from infancy to teens) and the nature of the syndrome as discussed above. Similarly there is the unexplained finding that, in mice, a FRa KO is not compatible with life, but in humans, the neurological manifestations do not occur until $\sim$ the second year of life. This delayed onset is not understood. This shows that in some cases $\mathrm{KO}$ mice are not necessarily accurate models of the human disease.

Another major problem is that the clinical pharmacology of the treatment of these transport disorders has not been studied adequately. (High arbitrary oral doses of $\mathrm{R}, \mathrm{T}$ or folates may not be optimal therapy but it is clear that at present early diagnosis and treatment even if suboptimal are critical before irreversible neurological occurs.) For example, in normal humans, Baker showed that $50 \mathrm{mg}$ of oral thiamine did not change the CSF total $\mathrm{T}$ ( $\mathrm{T}$ and TMP) concentration at six hours [54]. However, the same $50 \mathrm{mg} \mathrm{T}$ intravenously more than doubled the CSF total $\mathrm{T}$ concentration at 6 hours [54]. As noted above, in patients with autosomal recessive SLC52A2 transport disease, large oral doses of R ( 10 to $50 \mathrm{mg} / \mathrm{kg}$ per day) only increased the FAD blood levels by $35 \%$ [53]. I suspect that this is insufficient. I also hypothesize that treatment results would be better with an intravenous $\mathrm{R}$ regimen that needs to be defined e.g., with measurement of clinical outcomes. As noted above, less than $5 \%$ of total $\mathrm{T}, \mathrm{R}$ and folates normally enter brain by simple diffusion

As part of this discussion, I would ask: When are surrogate markers (e.g., folate concentrations in CSF) good enough or should clinical outcomes be the criterion to define the right dose as with the statins, not blood cholesterol? [55]. I favor the latter. Arguing by analogy, I note there are three treatments in clinical medicine that are almost magical: giving intravenous sugar to an insulin overdosed comatose diabetic, intravenous naloxone to a narcotic overdosed patient or intravenous thiamine to a Wernicke's encephalopathy patient with thiamine-deficient stupor/coma and paralysis of eye muscles. In all three cases "time is of the essence." Delayed or inadequate treatment (e.g., with oral thiamine) in the thiamine deficient patient described above can lead to the devastating Korsakoff's psychosis.

In summary, impressive progress has been made in the understanding of the vitamin transport disorders of folates, $\mathrm{T}$ and $\mathrm{R}$ (as opposed to deficiency disorders) with the ability to diagnose and treat and, in some cases, prevent these disorders. However, more work needs to be done - especially in defining the proper treatment regimens and doses. This work also shows that our understanding of genetic disorders of decades ago was too simple and in some cases misleading. In fact, the vitamin transport disorders of brain are heterogeneous and very complex in presentation and treatment but are now being unraveled at a molecular level and are another triumph of pediatric neurology.

\section{Acknowledgement:}

The author wishes to thank Michiko Spector for her aid in the preparation of this manuscript.

\section{References}

1. Davson H, Welch K, Segal MB (1987) The Physiology and Pathophysiology of the Cerebrospinal Fluid, New York, NY: Churchill Livingston.

2. Spector R, Lorenzo AV (1973) Ascorbic acid homeostasis in the central nervous system. Am J Physiol 225: 757-763.

3. Spector R, Lorenzo AV (1975) Folate transport by the choroid plexus in vitro. Science 187: 540-542.

4. Spector R, Lorenzo AV (1975) Folate transport in the central nervous system. Am J Physiol 229: 777-782.

5. Spector R (1977) Vitamin homeostasis in the central nervous system. N Engl J Med 296: 1393-1398.

6. Spector R (1976) Thiamine transport in the central nervous system. Am J Physiol 230: 1101-1107.

7. Spector R (1980) Riboflavin homeostasis in the central nervous system. J Neurochem 35: 202-209.

8. Spaan AN, Ijlst L, van Roermund CW, Wijburg FA, Wanders RJ, et al. (2005) Identification of the human mitochondrial FAD transporter and its potential role in multiple acyl-CoA dehydrogenase deficiency. Mol Genet Metab 86: 441-447.

9. McCarthy EA, Titus SA, Taylor SM, Jackson-Cook C, Moran RG (2004) A Mutation Inactivating the Mitochondrial Inner Membrane Folate Transporter Creates a Glycine Requirement for Survival of Chinese Hamster Cells. J. Biol. Chem 279: 33829-33836.

10. Lindhurst MJ, Fiermonte G, Song S, Struys E, De Leonardis F, et al. (2006) Knockout of Slc25a19 causes mitochondrial thiamine pyrophosphate depletion, embryonic lethality, CNS malformations, and anemia. Proc Natl Acad Sci U S A 103: 15927-15932.

11. Spector R, Coakley G, Blakely R (1980) Methionine recycling in brain: a role for folates and vitamin B-12. J Neurochem 34: 132-137.

12. Suleiman SA, Spector R (1980) Development and regional distribution of methionine synthetase in rabbit brain. J Neurochem 35: 1250-1252.

13. Suleiman SA, Spector R (1982) Metabolism of deoxyuridine in rabbit brain. J Neurochem 39: 824-830.

14. Zhao R, Diop-Bove N, Visentin M, Goldman ID (2011) Mechanisms of membrane transport of folates into cells and across epithelia. Annu Rev Nutr 31: 177-201.

15. Spector R, Johanson CE (2014) The nexus of vitamin homeostasis and DNA synthesis and modification in mammalian brain. Mol Brain 7: 3 .

16. Zhao R, Russell RG, Wang Y, Liu L, Gao F, et al. (2001) Rescue of embryonic lethality in reduced folate carrier-deficient mice by maternal folic acid supplementation reveals early neonatal failure of hematopoietic organs. J Biol Chem 276: 10224-10228.

17. Spiegelstein O, Mitchell LE, Merriweather MY, et al. (2004) Embryonic Development of Folate Binding Protein-1 (Folbp 1) knockout Mice: Effects of the Chemical Form, Dose, and Timing of Maternal Folate Supplementation. Developmental Dynamics 231: 221-231.

18. Jacobsen DW (2011) Knocking out the PCFT. Blood 117: 4683-4685.

19. Zhao R, Goldman ID (2013) Folate and thiamine transporters mediated by facilitative carriers (SLC19A1-3 and SLC46A1) and folate receptors. Mol Aspects Med 34: 373-385. 
20. McFarland R (2012) Cerebral folate deficiency--mishaps and misdirection. Brain 135: 2002-2003.

21. Steinfeld R, Grapp M, Kraetzner R, et al. (2009) Folate Receptor Alpha Defect Causes Cerebral Folate Transport Deficiency: A Treatable Neurodegenerative Disorder Associated with Disturbed Myelin Metabolism. Am. J. Human Genetics 85: 354-363.

22. Grapp M, Just IA, Linnankivi T, Wolf P, Lücke T, et al. (2012) Molecular characterization of folate receptor 1 mutations delineates cerebral folate transport deficiency. Brain 135: 2022-2031.

23. Ramaekers V, Sequeira JM, Quadros EV (2013) Clinical recognition and aspects of the cerebral folate deficiency syndromes. Clin Chem Lab Med 51: 497-511.

24. Cario H, Smith DEC, Blom H, Blau N, Bode H et al. (2011) Dihydrofolate Reductase Deficiency Due to a Homozygous DHFR Mutation Causes Megaloblastic Anemia and Cerebral Folate Deficiency Leading to Severe Neurologic Disease. Am. J. Human Genetics 88: 226-231.

25. Spector R, Johanson CE (2010) Choroid plexus failure in the KearnsSayre syndrome. Cerebrospinal Fluid Res 7: 14.

26. Spector R, Johanson CE (2007) Vitamin transport and homeostasis in mammalian brain: focus on Vitamins B and E. J Neurochem 103 425-438.

27. Reggiani C, Patrini C, Rindi G (1984) Nervous tissue thiamine metabolism in vivo. I. Transport of thiamine and thiamine monophosphate from plasma to different brain regions of the rat. Brain Res 293: 319-327.

28. Rindi G, Comincioli V, Reggiani C, Patrini C (1984) Nervous Tissue Thiamine Metabolism In Vivo. II. Thiamine and its Phosphoesters Dynamics in Different Brain Regions and Sciatic Nerve of the Rat. Brain Res 293: 329-342.

29. Patrini C, Reggiani C, Laforenza U, Rindi G (1988) Blood-brain transport of thiamine monophosphate in the rat: a kinetic study in vivo. J Neurochem 50: 90-93.

30. Dutta B, Huang W, Molero M, Kekuda R, Leibach FH, et al. (1999) Cloning of the human thiamine transporter, a member of the folate transporter family. J Biol Chem 274: 31925-31929.

31. Greenwood J, Love ER, Pratt OE (1982) Kinetics of thiamine transport across the blood-brain barrier in the rat. J Physiol 327: 95-103.

32. Lockman PR, Mumper RJ, Allen DD (2003) Evaluation of blood-brain barrier thiamine efflux using the in situ rat brain perfusion method. J Neurochem 86: 627-634.

33. Rindi G, Patrini C, Poloni M (1981) Monophosphate, the only phosphoric ester of thiamin in the cerebro-spinal fluid. Experientia 37: 975-976.

34. Reidling JC, Lambrecht N, Kassir M, Said HM (2010) Impaired intestinal vitamin B1 (thiamin) uptake in thiamin transporter-2-deficient mice. Gastroenterology 138: 1802-1809.

35. Boulware MJ, Subramanian VS, Said HM, Marchant JS (2003) Polarized expression of members of the solute carrier SLC19A gene family of water-soluble multivitamin transporters: implications for physiological function. Biochem J 376: 43-48.

36. Kevelam SH, Bugiani M, Salomons GS, Feigenbaum A, Blaser S, et al. (2013) Exome sequencing reveals mutated SLC19A3 in patients with an early-infantile, lethal encephalopathy. Brain 136: 1534-1543.

37. Tabarki B, Al-Shafi S, Al-Shahwan S, Azmat Z, Al-Hashem A, et al. (2013) Biotin-responsive basal ganglia disease revisited: clinical, radiologic, and genetic findings. Neurology 80: 261-267.
38. Spector R, Boose B (1979) Active transport of riboflavin by the isolated choroid plexus in vitro. J Biol Chem 254: 10286-10289.

39. Spector R (1980) Riboflavin transport in the central nervous system. Characterization and effects of drugs. J Clin Invest 66: 821-831.

40. Spector R (1981) Lumiflavin and lumichrome transport in the central nervous system. J Neurochem 36: 1186-1191.

41. Spector R (1980) Riboflavin accumulation by rabbit brain slices in vitro. J Neurochem 34: 1768-1771.

42. van Herwaarden AE, Wagenaar E, Merino G, Jonker JW, Rosing $\mathrm{H}$, et al. (2007) Multidrug transporter ABCG2/breast cancer resistance protein secretes riboflavin (vitamin B2) into milk. Mol Cell Biol 27: 1247-1253.

43. Yonezawa A, Masuda S, Katsura T, Inui K (2008) Identification and functional characterization of a novel human and rat riboflavin transporter, RFT1. Am J Physiol Cell Physiol 295: C632-641.

44. Yamamoto S, Inoue K, Ohta KY, Fukatsu R, Maeda JY, et al. (2009) Identification and functional characterization of rat riboflavin transporter 2. J Biochem 145: 437-443.

45. Yao Y, Yonezawa A, Yoshimatsu H, Masuda S, Katsura T, et al. (2010) Identification and comparative functional characterization of a new human riboflavin transporter hRFT3 expressed in the brain. J Nutr 140: 1220-1226.

46. Jusko WJ, Levy G (1975) Absorption, Protein binding and Elimination of Riboflavin. In: Riboflavin. Rivlin RS (ed). New York, NY Plenum Press.

47. Subramanian VS, Subramanya SB, Rapp L, Marchant JS, Ma TY, et al. (2011) Differential expression of human riboflavin transporters -1, -2, and -3 in polarized epithelia: a key role for hRFT-2 in intestinal riboflavin uptake. Biochim Biophys Acta 1808: 3016-3021.

48. Yonezawa A, Inui K (2013) Novel riboflavin transporter family RFVT/ SLC52: identification, nomenclature, functional characterization and genetic diseases of RFVT/SLC52. Mol Aspects Med 34: 693-701.

49. Bosch AM, Abeling NG, Ijlst L, Knoester H, van der Pol WL, et al. (2011) Brown-Vialetto-Van Laere and Fazio Londe syndrome is associated with a riboflavin transporter defect mimicking mild MADD: a new inborn error of metabolism with potential treatment. J Inherit Metab Dis 34: 159-164.

50. Johnson JO, Gibbs JR, Megarbane A, Urtizberea JA, Hernandez DG, et al. (2012) Exome sequencing reveals riboflavin transporter mutations as a cause of motor neuron disease. Brain 135: 2875-2882.

51. Bosch AM, Stroek K, Abeling NG, Waterham HR, Ijlst L, et al. (2012) The Brown-Vialetto-Van Laere and Fazio Londe syndrome revisited: natural history, genetics, treatment and future perspectives. Orphanet J Rare Dis 7: 83.

52. Nabokina SM, Subramanian VS, Said HM (2012) Effect of clinical mutations on functionality of the human riboflavin transporter-2 (hRFT-2). Mol Genet Metab 105: 652-657.

53. Foley AR, Menezes MP, Pandraud A, Gonzalez MA, Al-Odaib A, et al. (2014) Treatable childhood neuronopathy caused by mutations in riboflavin transporter RFVT2. Brain 137: 44-56.

54. Baker H, Thomson AD, Frank O, Leevy CM (1974) Absorption and passage of fat- and water-soluble thiamin derivatives into erythrocytes and cerebrospinal fluid of man. Am J Clin Nutr 27: 676-680.

55. Spector R, Snapinn SM (2011) Statins for secondary prevention of cardiovascular disease: the right dose. Pharmacology 87: 63-69. 